\title{
Escasez hídrica, género, y cultura mapuche. Un análisis desde la ecología política feminista
}

\author{
Leslie Bravo \\ Universidad de Chile, Santiago, Chile. \\ Email: leslie.bravo@ug.uchile.cl \\ Maria Christina Fragkou \\ Universidad de Chile, Santiago, Chile. \\ Email: mariac.fragkou@uchilefau.cl
}

\begin{abstract}
Resumen: Este artículo analiza los impactos que la escasez hídrica ha tenido sobre las prácticas cotidianas de uso de agua de mujeres mapuche en la Comuna de San Juan de La Costa, Chile. Se abordadesde la ecología política feminista, para analizar las desigualdades hídricas que se experimentan a nivel doméstico en función del rol de género y la cultura de usuarios de agua. Se utiliza una metodología mixta, a través de la aplicación de encuestas, entrevistas y grupos focales.Se obtiene como resultado, que los impactos que la escasez hídrica genera sobre las mujeres mapucheson particulares, afectando su economía, emocionalidad y espiritualidad de maneras diferenciadas en función del tipo y fuente de acceso de que dispongan. Concluimos que los impactos domésticosde la escasez hídrica son heterogéneos, por lo que es relevanteanalizar los efectos microescalares producidos por las desigualdades socioambientales.
\end{abstract}

Palabras Claves: Sequía; ecología política; geografía de género; mujeres mapuche.

\section{Water scarcity, gender, and Mapuche culture. An Analysis from Feminist Political Ecology}

\begin{abstract}
This article analyzes the impacts that water scarcity has had on the daily water use practices of Mapuche women in the Commune of San Juan de La Costa, Chile. It is approached from the feminist political ecology, to analyze the hydric inequalities that are experienced at domestic level in function of the gender role and the culture of water users. A mixed methodology is used, through the application of surveys, interviews and focus groups.As a result, the impacts that water scarcity generates on Mapuche women are particular, affecting their economy, emotionality and spirituality in different ways depending on the type and source of access they have. We conclude that the domestic impacts of water scarcity are heterogeneous, so it is relevant to analyze the microscale effects produced by socio-environmental inequalities.
\end{abstract}

Keywords: Drought, political ecology, gender geography, mapuche women.

\section{Escassez de água, gênero, e cultura mapuche. Uma análise da ecologia política feminista}

Resumo: Este artigo analisa os impactos da escassez de água nas práticas cotidianas de uso da água das mulheres mapuche na Comuna de San Juan de La Costa, Chile. Aborda-se a partir da ecologia política feminista, para analisar as desigualdades hídricas que são vivenciadas no nível doméstico em termos do papel do gênero e da cultura dos usuários de água. Uma metodologia mista é utilizada, através da aplicação de pesquisas, entrevistas e grupos focais.Obtém-se como resultado, que os impactos que a escassez de água gera sobre as mulheres mapuche são particulares, afetando sua economia, emotividade e espiritualidade de formas diferenciadas dependendo do tipo e fonte de acesso disponível. Conclui-se que os 
impactos domésticos da escassez de água são heterogêneos, por isso é relevante analisar os efeitos de microescala produzidos pelas desigualdades socioambientais.

Palavras-chaves: Seca;ecologia política; geografia de gênero; mulheres mapuche.

$$
* * *
$$

\section{Introducción}

El agua en territorios rurales es un componente que históricamente ha sido de difícil acceso para su población. Así se tiene que para el año 2017, un 47,2\% de la población rural en Chile no contaba con abastecimiento formal de agua potable (Fundación Amulén, 2019). Esto, debido a la fragilidad que la disponibilidad de este elemento tiene frente a diversos factores geográficos, demográficos, económicos y políticos (Swyngedouw, 2009). Los territoriosrurales presentan dificultades por dispersión geográfica y falta de infraestructura hidráulica, mientras que también es posible identificar dificultades económicas y/o sociales para acceder a fuentes y formas de organización comunitaria, que faciliten el abastecimiento de agua potable de manera estable y segura (Fundación Amulén, 2019). Sumado a esto, se presenta la presión sobre la disponibilidad de agua generada en territorios turísticos, frente al aumento demográfico en temporadas estivales (Rico, 2009), mientras que los niveles de precipitaciones desde la mitad de siglo XX han estado descendiendo (Figueroa, 2014).

Adicionalmente, la situación se intensifica frente al aumento de sistemas de producción extractivista como es el caso de la industria forestal que destaca en extensión dentro de los territorios rurales del sur de Chile (INE, 2007), y que, de acuerdo a Catalán et al. (1999),Otero (1997) yTorres-Salinas et al. (2016)disminuyen los caudales de aguas lluvias que infiltran en el suelo provocados por un incremento en la tasa de evapotranspiración, lo que genera consecuentemente disminución en los acuíferos; empeorando de esta manera el paisaje hídrico de los territorios rurales.

En este artículo, argumentamos que los cambios socioambientales que experimentan los territorios rurales, tienen alto impacto en la disponibilidad y calidad de las fuentes hídricas que abastecen a la población habitante de estos sectores, quienes a menudo dependen de fuentes frágiles, dispersas, e informales para abastecerse de agua. A su vez, este cambio en la disponibilidad hídrica impacta de manera directa en el tipo de abastecimiento a nivel doméstico, del cual se hacen cargo principalmente las mujeres (Stratford, 1995).Dado que son las mujeres las encargadas del espacio doméstico, son ellas mismas quienes se ven impactadas sobre sus labores y en consecuencia se ven obligadas a adaptarse, proponer estrategias y a buscar soluciones ante la falta de agua (Truelove, 2011).Por ello, nuestro estudio se enfoca en analizar los impactos que la escasez hídrica ha tenido sobre la vida cotidiana de mujeres mapuche habitantes de la comuna de San Juan de La Costa, tomando en cuenta que son ellas quienes, ya sea por cuestión de género y/o factores culturales, se encargan de la economía del hogar, relacionándose directamente con el agua para llevar a cabo las labores de base del espacio doméstico.

Por consiguiente, basándonos en el marco teórico de la ecología política feminista (EPF), se propone que la escasez hídrica se encarna de maneras diferenciadas según rasgos culturales, sociales, y de género(Rocheleau et al., 2015). En este sentido, el territorio estudiado es de interés debido a que se trata de un territorio que además de ser rural, presenta amplia concentración de población perteneciente a la cultura mapuche huilliche, que se caracteriza por tener una cosmovisión que da relevancia a la relación con la naturaleza y sus recursos. Estos aspectos sociales y culturales, permitirán demostrar las diferentes formas en que los modos de vida y las cotidianidades desarrollados en elterritorio se ven impactadas por la falta de agua.

Este artículo busca indagar en cuestiones poco exploradas dentro de los estudios socioambientales a nivel nacional, como son las dinámicas hídricas dentro de espacios domésticos, las estrategias de acceso y uso de agua en áreas rurales, y los impactos que la escasez hídrica tiene para las mujeres mapuche en términos físicos, emocionales y espirituales, alumbrando de esta manera nuevos aspectos de los impactos de la escasez hídrica entre las diferentes identidades y el medioambiente. 
Para llevar a cabo esta investigación, se utilizó una metodología mixta, a través del análisis de aspectos cuantitativos y cualitativos. Para ello se realizó un primer trabajo de campo entre los meses de Septiembre y Noviembre del año 2016, donde se accedió a la percepción de la población respecto a los cambios cuantitativos y cualitativos que han experimentado las fuentes hídricas, a través de encuestas que posteriormente fueron sistematizadas por el software SPSS. Posteriormente, se realizó una segunda visita a terreno, durante el mes de Junio del año 2017, para llevar a cabo entrevistas y grupos focales, las cuales fueron analizadas a través del software Atlas.ti, que permitiesen conocer cómo el cambio de disponibilidad y calidad del agua de las diversas fuentes impacta sobre las vidas cotidianas de las mujeres mapuche de la comuna.

Nuestros resultados demuestran que, por un lado, los cambios en las fuentes de abastecimiento nos son homogéneas (Sultana, 2015), más bien, dependen de aptitudes geográficas, económicas y sociales; y por otro lado, los impactos que se producen a niveles domésticos dependen de las situaciones particulares que encarnen las mujeres mapuche estudiadas.

A continuación se presenta el marco teórico que da sostén a esta investigación, el cual toma como eje la propuesta de la EPF, que nos ayuda indagar en la cotidianidad de las mujeres rurales, y asociarla a la disponibilidad de las fuentes hídricas. Luego se presenta el caso de estudio, para posteriormente ahondar en los resultados obtenidos y finalizar con las principales discusiones y conclusiones del estudio.

\section{La ecología política feminista como marco de análisis}

La EPF nace como campo de estudio a raíz de la necesidad de establecer un puente de unión entre las investigaciones de la ecología políticaque realizan análisis macroescalares, y la necesidad de profundizar en las dinámicas que las desigualdades socioespaciales generan a nivel microescalar(Truelove, 2011). Para comprender la relación entre ambos paradigmas, se abordarán en primera instancia, las aplicaciones que la ecología política ha propuesto para comprender las relaciones que los territorios tienen con el agua, con el fin de indagar posteriormente sobre los aspectos que profundiza la EPF.

La ecología política del agua, parte de la premisa que las desigualdades socioambientales esconden una serie de relaciones de poder que son dadas bajo un contexto político específico (Leff, 2006; Prieto, 2006). Logrando de esta manera, desnaturalizar las desigualdades hídricas y por contraparte, politizarlas (Prieto, 2006). Desde allí, se plantea que la forma en que se gestiona, accede, y usa el agua estará influida a la vez por condiciones climáticas y geográficas, pero además por cuestiones sociales, políticas y económicas, propias de cada territorio (Swyngedouw, 2009).

Por lo tanto, a través de la lectura realizada por la ecología política de las relaciones entre los recursos hídricos y las sociedades, se cuestiona el análisis clásico del "Ciclo Hidrológico" que conceptualiza el agua como un elemento químico, neutro de intervenciones sociales (Linton, 2014), para proponer el concepto de "Ciclo Hidrosocial" (Linton, 2014; Swyngedouw, 2009). El ciclo hidrosocial hace referencia a la influencia mutua que tienen el agua y la sociedad, señalando que, a la vez que el agua condiciona las capacidades humanas de acceso y uso de agua, serán las mismas relaciones humanas, y sus dinámicas sociales, económicas y políticas, las que modifiquen y condicionen la disponibilidad de agua en los territorios (Budds et al., 2012). Generándose de esta manera, un ciclo que se hace y rehace entre el comportamiento y la organización social y los flujos hídricos (Budds et al., 2012).

Por lo anterior, al analizar situaciones de desigualdad hídrica, toma especial relevancia poner atención al comportamiento que las poblaciones y sus relaciones sociopolíticas tienen frente al recurso hídrico; cuestión que ha sido bien atendida por la tradición de la ecología política. Sin embargo, como señala Truelove (2011),

los análisis de la ecología política se basan en atender los procesos socioambientales que producen desigualdades de acceso y uso de recursos, enfocándose principalmente en los aspectos de clase que producen estas desigualdades a niveles macroescalares. 
Pese a ello, los análisis macroescalares no logran captar los procesos individuales y la forma en que la sociedad encarna en sus vidas cotidianas las desigualdades socioambientales (Truelove, 2011). Por lo que se tiende a generalizar y homogeneizar realidades al no abordar las realidades hídricas particulares desde una lente más fina (Sultana, 2015). Por lo tanto, es en la necesidad de ahondar en aspectos microescalares, que se propone el paradigma de la EPF para estudiar cómo las desigualdades hídricas se manifiestan en los espacios domésticos (Truelove, 2011).

El paradigma de la EPF nace a partir de la fusión de diferentes conceptualizaciones que plantea la crítica feminista -a partir de las que se cuestionan las bases epistemológicas que construyen las relaciones de la humanidad con el entorno. Para ello se toman aspectos del ecofeminismo, la economía ecológica, la economía feminista, el feminismo socialista y el feminismo posestructuralista; para posteriormente aplicarlas dentro de la línea de la investigación geográfica (Rocheleau et al., 1995). Desde allí, se abordan aspectos particulares como el género, la raza, la diversidad cultural, entre otros; así como las relaciones de poder dentro del hogar (micropolíticas), las prácticas domésticas, y las corporalidades y subjetividades, con el fin de analizar desde nuevas perspectivas los estudios socioambientales (Rocheleau et al., 1995).

Cabe mencionar, que en un sentido metodológico, la EPF, puede ser útil para identificar y analizar las pautas cotidianas que encarna cada individuo a raíz de las dificultades generadas por el desigual acceso a los recursos. Ya que, generalmente los análisis macroescalares de los conflictos socioterritoriales "ven a las comunidades como entidades homogéneas, por lo que pueden pasar por alto realidades complejas donde el acceso y el control sobre los recursos hídricos varían a través de múltiples sistemas de diferenciación entrelazados y jerárquicos” (Sultana,2015, p.261).

Por lo anterior, en este estudio se abordan las categorías de género y cultura, entendiendo el género como una construcción social (Lamas, 2000) que atribuye a unos (hombres) el espacio masculino-productivopúblico y a otras (mujeres) el espacio femenino-reproductivo-privado. Ya que, es así como se define a "uno y otro sexo en materia de moral, psicología y afectividad. La cultura marca a los sexos con el género y el género marca la percepción de todo lo demás: lo social, lo político, lo religioso, lo cotidiano” (Lamas, 2000, p.4). De esta manera, se busca analizar las prácticas que encarna la mujer mapuche rural de San Juan de La Costa, en tanto es ella quien maneja la base de la reproducción de la vida, lo que la posiciona como encargada de la crianza, los cuidados, la transmisión de la cultura, la agricultura y ganadería de subsistencia, y la artesanía, entre otros roles (Quilaqueo, 2013). Situación que genera que la capacidad que las mujeres tengan de acceder al recurso hídrico, será lo que facilitará o dificultará la vida cotidiana de estas.

De aquí se desprende que, situaciones de desigual distribución de agua, además de provocar efectos diferenciados en los grupos de acuerdo a su clase social, estos se vivenciarán desigualmente también a escalas particulares; los que dependerán a su vez de la raza, la cultura, la edad, el género -y una serie de aspectos que conforman la identidad de cada individuo - siendo, por lo tanto encarnados de forma única en cada cuerpo.Cabe destacar que los efectos físicos no son los únicos que se impregnan en los cuerpos al carecer del acceso a recursos vitales como el agua, sino más bien se entrelazan una serie de sentimientos y cargas emocionales en la búsqueda por el acceso a esta; como lo señala Sultana (2011):

"la vida cotidiana no se trata solo de obtener suficientes recursos, ya que las luchas para lograr esos recursos cobran un precio y complican las vidas emocionales y materiales de las mujeres y sus familias. Esto tiene una relación directa con las formas en que se accede, usa y lucha el agua en una localidad” (p.165).

En resumen, la EPF, al considerar “el género como una variable crítica que conforma el acceso de los recursos, y su control, al interactuar con la clase, la casta, la raza, la cultura y la etnicidad para, así dar forma a procesos de cambio ecológico”(Rocheleau et al., 1995, p. 345), permite visibilizar las dinámicas que históricamente han realizado las mujeres dentro de los espacios domésticos, y comprender cómo estas resisten y sobreviven cotidianamente en contextos de escasez hídrica. 


\section{Comuna de San Juan de La Costa; territorio en emergencia hídrica}

El caso de estudio comprende la comuna de San Juan De La Costa, perteneciente a la provincia de Osorno, en la X Región de Los Lagos. Esta comuna tiene una superficie de $1.317 \mathrm{~km}^{2}$ que forma parte del 2,36\% de la superficie regional (PLADECO, 2012). El territorio es mayoritariamente rural con una población de 7.000 habitantes, de los cuales un $90 \%$ pertenecen a la cultura mapuche huilliche.

En términos económicos, esta comuna presenta un índice de pobreza de 12,2\%, lo que la posiciona por debajo de la tasa de pobreza nacional, siendo por ello una de las comunas rurales más vulnerables de Chile (PLADECO, 2012). Dentro de las actividades económicas que se llevan a cabo, se encuentran la pesca artesanal, el turismo, la artesanía, la agricultura y la ganadería, y como la principal se posiciona la actividad de explotación forestal; actividad que toma fuerza en el año 1974, a través del decreto de Ley Nº701 de Fomento Forestal (Camus, 2006). Esto, sumado a la poca aptitud agrícola de los suelos de la comuna (altas pendientes, baja materia orgánica, poca permeabilidad y retención de aguas), potencia el establecimiento de sistemas de producción forestal (DGA, 2004). Lo que ha generado que del total de la superficie regional forestada, la comuna de San Juan de la Costa concentre un 9\%, de lo cual un 35\% corresponde a las especies exóticas de Eucaliptus Nitens y un 63\% a Pino Radiata y Oregón, mientras que en un 2\% se encuentran plantaciones de especies como Álamo, Aromo, Raulí, Coigüe, entre otros(INE, 2007). Situación que, tomando en cuenta lo estudiado, explicaría un vínculo existente entre el cambio extensivo en el uso de suelo -particularmente hacia plantaciones de monocultivo forestal-, con la disminución de la disponibilidad del recurso hídrico en el territorio (Otero et al., s/f; Camus, 2006).

Geográficamente, esta comuna se emplaza sobre dos geoformas: La cordillera de la costa y las planicies litorales. Por una parte, se encuentra la Cordillera de la Costa, que se presenta como un cordón montañoso cuya altura trasciende sólo en algunos puntos los 600 m s.n.m. (BCN, s/f), y cuyas características generan alta dispersión demográfica. Por otra parte, se encuentra la Planicie Litoral, la que está limitada por pequeñas playas interrumpidas por acantilados que proceden de la cordillera de la costa (BCN, s/f) tales como: Bahía Mansa, Pucatrihue y Maicolpué; territorios que junto a la localidad de Puaucho comprenden los núcleos de mayor concentración de población del territorio. La comuna posee un clima templado cálido lluvioso con influencia mediterránea, cuya característica principal era manifestar precipitaciones todo el año, presentando menos pluviosidad en verano (DGA, 2004). Sin embargo, en el periodo estudiado, la comuna experimentó una disminución en la cantidad de precipitaciones, situación que se intensificó para el año 2013, temporada en que la población manifestó la sequía de pozos y vertientes que les abastecían, declarándose la comuna en estado de “Emergencia Hídrica”. Desde allí, se han tomado una serie de medidas desde las distintas instituciones encargadas del abastecimiento hídrico, que buscan paliar el problema, siendo la de más rápida respuesta el abastecimiento de agua potable mediante camiones aljibes, -situación que al año 2017, mantenía operando siete camiones aljibe alrededor de la comuna-.

Con posterioridad a este evento, en el año 2015, la Subsecretaría de Agricultura declara en "Emergencia Agrícola” a un conjunto de regiones, dentro de las que se hallaba comprometida la Región de los Lagos en su totalidad. Esta situación fue atribuida a las condiciones de "sequía", que provocaron una serie de impactos en el ámbito de la producción agrícola para los sectores afectados (BCN, 2015).

Los factores anteriormente mencionados han generado fuertes tensiones sobre el recurso hídrico del territorio, impactando trascendentalmente sobre las dinámicas históricas de abastecimiento hídrico y cambiando consecuentemente las fuentes hídricas a las que accede la población en sus espacios domésticos, lo que se presenta a continuación.

\section{Historicidad de Fuentes y Tipos de abastecimiento hídrico}

Para ordenar los resultados de esta investigación el territorio de San Juan de La Costa se dividirá, tomando como límite las dos geoformas que le componen: la Cordillera de la Costa, y las Planicies Litorales. Ambos territorios se han visto impactados por la falta de disponibilidad de agua de manera diferenciada, por lo que se identificaron las pautas de acceso hídrico históricas y posteriores a los episodios de escasez para cada territorio. 
La Cordillera de la Costa se presenta como un territorio rural con alta dispersión demográfica. Debido a ello, este sector históricamente ha utilizado estrategias informales, particulares y/o comunitarias, para acceder al agua, tales como extracción de agua directamente de ríos, esteros, vertientes y pozos o norias. En este territorio, el acceso a agua potable no solía ser un problema, ya que poseían fuentes que debido a la infiltración subterránea del agua, se percibían como confiables por la población. Además el acceso a una u otra fuente dependía de la distancia hacia aquellas fuentes naturales, y de la capacidad económica que cada familia tuviera para utilizar estrategias tecnológicas como cañerías, pozos profundos, bombas de impulsión, entre otras.

Por contraparte, las Planicies Litorales se presentan como el territorio que posee mayor concentración de población. Por ello, a partir de las políticas de desarrollo que impulsaron la potabilización del agua en territorios rurales (Villarroel, 2012), se establecen los Comités de Agua Potable Rural (CAPR) de Bahía Mansa (1980), Pucatrihue (1993) y Maicolpue (1996), junto a Puaucho (1998), la localidad central que concentra el polo administrativo.

De manera que, para estos territorios, el establecimiento de CAPR, generó la facilidad de acceder al agua a través de un sistema de red de cañerías que extraen el recurso hídrico kilómetros aguas arriba, el que posteriormente es tratado químicamente para entregarlo en el hogar de cada familia. Cabe destacar que en este mismo territorio han convivido habitantes que no se encuentran adheridos al sistema de Agua Potable Rural, los que se abastecían principalmente de agua de vertientes, pozos y ríos (en algunos casos, dejando de lado en otros, la extracción directa de agua de río debido a la percepción de contaminación por aumento demográfico en la rivera de los ríos). Sin embargo, a partir del año 2013, estas dinámicas que parecían mantener el acceso y uso de agua estable para los habitantes de cada territorio, se vieron impactadas por la fuerte disminución de agua que experimentaron las fuentes de mayor fragilidad hídrica: pozos y vertientes de ambos territorios comenzaron a manifestar fuertes disminuciones, al punto de experimentar la sequía completa en los casos más extremos. Este comportamiento generó fuertes tensiones en el territorio, por lo que la institucionalidad puso en pie una serie de medidas para compensar la dificultad de abastecimiento.

\section{Territorio en Escasez: Impacto sobre las formas de acceso de agua}

El territorio de la Cordillera de la Costa durante el periodo de estudio es el sector que presentó la mayor cantidad de población afectada por la falta de agua. Un aproximado de 500 familias recibe agua potable suministrada a través de camiones aljibe (la cual es entregada por medio de la ONEMI y el Municipio de San Juan de La Costa). De acuerdo a la población, la disminución de agua en las fuentes naturales que históricamente les abastecieron, ha tenido un fuerte impacto sobre sus cotidianos, yaque en el periodo mencionado el total de la población encuestada dependía del suministro de agua entregado por camiones aljibe (la entrega de agua se efectuaba una vez por semana y constaba de una cantidad aproximada a 50 litros diarios por persona). Cabe destacar que esta situación, además de generarles la dependencia de un abastecimiento discontinuo y de fuente externa a sus territorios, en algunos casos les genera la incertidumbre de no poder contar con el suministro en periodos de pluviosidad intensa, en que algunos de los afectados no logran ser abastecidos, debido a irregularidades topográficas que condicionan la estabilidad del terreno, imposibilitando el paso a los camiones.

Debido a esta dificultad, y a la limitada cantidad de agua que se les entrega, la población cuenta además con otras estrategias de acceso al recurso, utilizando de manera paralela otras fuentes. Así, se tiene que la mayor parte de la población utiliza agua de pozo, en la medida que el estado de este es adecuado, la que es utilizada principalmente para consumo humano y doméstico. El agua de esteros y vertientes se utiliza en menor medida, ya sea por percepción de contaminación de estas fuentes (que atribuyen al uso de pesticidas forestales) y/o por la lejanía. Sin embargo, cabe destacar que al no obtener agua potable de camión aljibe -por las razones anteriormente mencionadas- la población se ve muchas veces en la necesidad de desplazarse en busca de agua. Es importante mencionar que la recopilación de aguas lluvias se presenta como una nueva estrategia, las que a su vez se reutilizan para ciertas actividades (así explican, por ejemplo, que en algunos casos el agua de lavado es utilizada posteriormente para riego de huerto). 
Por otra parte, en el territorio Litoral la percepción de impacto de la escasez hídrica es más débil entre la población, debido a la mayor estabilidad del sistema de Comités de Agua Potable Rural, a la que gran parte de los habitantes del territorio logran acceder, y que cuenta con fuentes que sólo evidencian disminuciones en periodos estivales atribuidas a la disminución de los caudales de ríos y esteros que abastecen desde aguas arriba y al aumento demográfico. Por esta situación, en algunos puntos del territorio se les suministra agua de camiones aljibes en estanques comunitarios, que tienen mayor capacidad de almacenamiento (10.000 litros), la que deben distribuir de manera autoorganizada entre vecinos. En este mismo territorio se encontraron grupos de familias que no pertenecían alos CAPR debido a su ubicación geográfica, quienes sí reconocieron complicaciones sobre las fuentes que históricamente les abastecieron y a cambio debieron depender del agua suministrada por camiones aljibes. Además, se encontró el caso de comunidades mapuche que contaban con sistemas de abastecimiento comunitarios informales que solían ser gestionados por sus mismas organizaciones, quienes al ver desestabilizadas las fuentes de estero y vertiente, debieron acceder a sistemas más sofisticados, subvencionados por la institucionalidad, como son los Estanques Australianos. Este sistema es gestionado a través de INDAP y/o CONADI, y permite acumular mayores cantidades de aguacon fines agrícolas; sin embargo,esta agua es utilizada por la población en función de sus necesidades, pudiendo ser igualmente destinadapara consumo directo.

Es importante destacar que, en este territorio, pese a no expresarse de manera masiva la disminución hídrica, existe un discurso entre las y los encuestados que plantea la problemática hídrica como una crisis, frente a la que mencionan utilizar estrategias de reutilización de aguas, y en algunos casos recopilación y almacenamiento de aguas lluvias, como medida de resguardo frente a potenciales episodios de escasez, ya sea por falla del sistema de APR y/ o falta de disponibilidad de agua de camión aljibe.Con el fin de sintetizar los diferentes tipos y fuentes hídricas de las que depende la población de San Juan de La Costa, en el siguiente cuadro se identifican las características principales asociadas a la forma de acceso de los usuarios para cada una de ellas (Cuadro 1).

\section{Cuadro 1 \\ Características de formas acceso por tipo y fuente de abastecimiento hídrico.}

Fuente: Elaboración propia en base a síntesis de discursos analizados

\begin{tabular}{|c|c|c|}
\hline \multicolumn{2}{|r|}{$\begin{array}{l}\text { Tipos y fuentes de } \\
\text { abastecimiento }\end{array}$} & \multirow{2}{*}{$\begin{array}{l}\text { Caracteristicas de formas de acceso para los usuarios } \\
\text { - Se entrega semanalmente directamente en la vivienda } \\
\text { - Suministro Discontinuo } \\
\text { - Se entrega una cantidad limitada de agua (50 litros diarios por } \\
\text { persona). } \\
\text { - Los camiones no pueden ingresar a todos los territorios }\end{array}$} \\
\hline \multirow{4}{*}{ 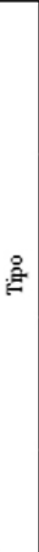 } & Camion Aljibs & \\
\hline & $\begin{array}{c}\text { Agua Potable Rural } \\
\text { (APR) }\end{array}$ & $\begin{array}{l}\text { - Es entregada directamente en la vivienda } \\
\text { - Suministro Continuo } \\
\text { - Sufre cortes por desgaste de infraestructura } \\
\text { - Sufre disminución en periodos estivales por sumentos } \\
\text { demográficos. }\end{array}$ \\
\hline & $\begin{array}{l}\text { Agua aimacenada } \\
\text { en Estanques } \\
\text { comwitarios }\end{array}$ & $\begin{array}{l}\text { - Permiten almacenar mayor cantidad de aguas (lluvisa o entregada } \\
\text { por camión aljibe) } \\
\text { - Suministro Discontinuo } \\
\text { - Pusden generar desigualdad de acceso entre las familias }\end{array}$ \\
\hline & $\begin{array}{l}\text { Agua aimacenada } \\
\text { en Estangues } \\
\text { anistralianos }\end{array}$ & $\begin{array}{l}\text { - Permiten almacener agua de manera permanente } \\
\text { - Suministro Continuo } \\
\text { - Deqenden de la concentración de población }\end{array}$ \\
\hline \multirow{4}{*}{ 兽 } & Agua de Pozo & $\begin{array}{l}\text { - Absatece directamente en el hogar } \\
\text { - Suministro Continuo } \\
\text { - Son la fuente mas impactsda por la escasez } \\
\text { - Sufren disminución y sequia completa en algunos casos }\end{array}$ \\
\hline & $\begin{array}{c}\text { Agua de rioo } \\
\text { estero }\end{array}$ & $\begin{array}{l}\text { - Disponen de diferentes puntos para extraer el agua } \\
\text { - Suministro Continuo } \\
\text { - Daben recorrer largas distancias con baldes o carretas } \\
\text { - Acuden varias veces a la semana } \\
\text { - Seperciben con desconfianza por contaminación }\end{array}$ \\
\hline & Aguar linvias & $\begin{array}{l}\text { - Se puede acceder tras cada periodo de lluvia } \\
\text { - Se almacena agua en cualquier tipo de recipiente } \\
\text { - Suministro Discontinuo } \\
\text { - Su disponibilidad depende del clima } \\
\text { - Tiene un límite de tiempo de uso (posteriomente cambian las } \\
\text { propiedades organolépticas del agua) }\end{array}$ \\
\hline & Agua de vertiente & $\begin{array}{l}\text { - Es agua percibida como "limpia" por la población } \\
\text { - Se debe salir del espacio doméstico pera acceder a ella } \\
\text { - A veces recorrer grandes distancias } \\
\text { - El caudal se puede reducir en periodos de sequía }\end{array}$ \\
\hline
\end{tabular}




\section{Escasez hídrica y vida cotidiana de las mujeres mapuche}

A partir del análisis de los impactos que la escasez hídrica en San Juan de La Costa ha tenido sobre las dinámicas de acceso hídrico, fue posible indagar sobre los usos que las mujeres le dan cotidianamente al agua, indagando de esta manera dentro de los impactos materiales y emocionales que la falta de disponibilidad de agua ha generado en su vida diaria.

Para ello, se realizaron tres grupos focales alrededor del territorio. A través de estos, se realizaron preguntas abiertas para conocer las condiciones hídricas del espacio que habitaba cada mujer, la relación que mantenían con el agua, sus percepciones respecto a la escasez hídrica en el territorio; para finalizar con impresiones más personales respecto a la afectación de sus vidas cotidianas y emocionalidades en el contexto de crisis hídrica que enfrentaban.

Por esto, se realizaron dos reuniones en el territorio Litoral. Allí se entrevistó a cinco mujeres de la comunidad mapuche Maicolpi y cinco de la agrupación indígena Mapulafken, cuyo rango etariofluctuaba entre los 35 y 65 años. Por otra parte, en el territorio Cordillerano, se realizó un encuentro con la Agrupación de Mujeres Emprendedoras de San Juan de La Costa. Allí, se entrevistó a 10 mujeres mapuche, habitantes de distintos sectores de este territorio, cuyas edades se situaron entre los 40 y 70 años.

Acontinuación,se presenta una síntesis de los usos principales, las formas de recepción y distribución del agua dentro del espacio doméstico y las estrategias que las mujeres utilizan para conservar y/o proteger el recurso hídrico en sus territorios.

Las mujeres mapuche de la comuna de San Juan de La Costa llevan a cabo dos roles dentro del espacio doméstico. Por una parte, todas las entrevistadas reconocieron hacerse cargo de la gestión de todos los trabajos domésticos efectuados dentro del hogar (alimentación, limpieza, crianza y cuidados de familiares enfermos), además de -la gran mayoría- encargarse de actividades de subsistencia económica que dependen tanto del espacio doméstico, como del espacio público para poder ser llevadas a cabo (agricultura, artesanía en lana, ganadería en pequeña escala y crianza de aves, y turismo -para el caso de aquellas que habitan el territorio litoral-).

En este punto es importante notar que cada una de estas actividades se encuentra estrechamente vinculada y es dependiente del acceso al recurso hídrico; desde las actividades de base para el sostenimiento de la vida, hasta aquellas que traspasan los límites domésticos para transformarse en actividades económicamente productivas. Por ello, cabe destacar, que las estrategias que cada mujer deberá gestionar dependerán a su vez de las fuentes de agua con las que cuenten, de las actividades que deban realizar, de la ayuda que obtengan (económica y físicamente), y la disponibilidad de agua que haya en su territorio.

Así, se obtiene que las mujeres habitantes de la Cordillera de la Costa se hayan visto mayormente afectadas por la falta de disponibilidad de agua. En este territorio, las mujeres son las principales encargadas de la recepción y posterior distribución del agua dentro del espacio doméstico (de acuerdo a las encuestas en un 68\% se hace cargo de esta labor la mujer, en un $18 \%$ la familia completa y en un $14 \%$ el hombre). Ellas mencionan sentir un alto grado de dependencia de la entrega de agua de camión aljibe, lo que las sitúa en un lugar de impotencia frente a la realización de sus actividades diarias. Por esta razón, deben priorizar unas actividades por sobre otras: dando mayor importancia a las actividades de consumo y limpieza, seguidas por el agua para agricultura, lo que se explica debido a que gran parte de la población de este territorio vive de la agricultura de subsistencia -para alimentación propia o para comerciar fuera del territorio-; dejando de lado en algunos casos actividades históricas como la artesanía en lana, la agricultura de subsistencia y la ganadería. Pese a ello, aparecen experiencias en que las mujeres que no dejan de lado sus actividades, eligen recorrer una mayor distancia en busca de agua de estero o vertiente para lavar ropa y/o lana.

Cabe destacar dentro de este mismo territorio estrategias de reutilización hídrica tales como la recopilación de aguas lluvias, la distribución cuidadosa del agua para cada actividad y la posterior reutilización del agua para actividades como el huerto y/o estanques de baño. Por otra parte, en este territorio se entrelazan las percep- 
ciones respecto a las propiedades organolépticas de las fuentes hídricas. Encontrándose mujeres que confían de las fuentes de agua potable debido al tratamiento químico que prevén en estas, sin embargo no gustan del sabor. Mientras que otra parte prefiere aún utilizar fuentes hídricas naturales como aguas de pozos y vertientes debido al sabor, las que reconocen hervir antes de consumir por desconfianza frente a la potencial contaminación por actividades forestales. Por lo que la fuente hídrica que finalmente utilicen para una u otra actividad dependerá de las percepciones que la población -y en particular las mujeres- tengan de la calidad del agua.

Para el caso de las Planicies Litorales, el panorama hídrico que viven las mujeres mapuche es distinto. Esto, debido a que la concentración de Comités de Agua Potable Rural resulta en que la población no perciba la disminución de agua de manera directa, por lo que los impactos sobre la vida cotidiana de estas mujeres son menos críticos. La mayoría de las que se encuentran adheridas a CAPR, perciben el acceso a agua como seguro, y tienen acceso directo y diario, exceptuando los periodos de disminución de caudal en temporadas estivales, momento en que las estrategias de acceso y uso cambian: la población (en este caso es una labor que en un $60 \%$ realiza la familia, en un $25 \%$ la mujer y un $15 \%$ el hombre) va en busca de agua a las vertientes cercanas, y/o utilizan agua lluvias almacenadas con antelación.

Lo mismo se plantea para el caso de comunidades mapuche que autogestionan sus fuentes de abastecimiento: la labor de recopilación de agua se distribuye entre los diferentes integrantes del grupo familiar. Sin embargo, es importante distinguir que pese a que en este territorio, ya sea por cuestiones de densidad demográfica y/o nivel de urbanización, la labor de recopilación y búsqueda de agua la realiza la familia de manera indistinta, son las mujeres quienes se hacen principalmente cargo de la decisión de qué agua usar, cuánta, y para qué labor. Así se tiene que el agua en este territorio es mayormente utilizada para consumo humano y limpieza, y a diferencia del territorio Cordillerano, su uso productivo es prioritario para actividades turísticas (hospedería, restaurantes, y elaboración de artesanía). Situación por la cual las mujeres identifican la merma de agua como una amenaza directa para realizar la actividad turística. Asimismo, en este territorio se llevan a cabo estrategias como recopilación de aguas lluvias, reutilización de aguas y el abastecimiento de casas familiares y vecinas.

Finalmente, es importante destacar que aparejadas con las estrategias de reutilización y cuidado del recurso hídrico, las mujeres -sin distinción territorial- establecen una serie de vínculos entre la conservación del bosque nativo y la disponibilidad de agua, a la vez que atribuyen la merma de agua a la plantación extensiva de monocultivo forestal. A partir de esta percepción, han tomado acciones como la tala de árboles de pino y eucalipto de sus territorios y por contraparte la reforestación de árboles nativos en los mismos.

\section{Impactos de la escasez de agua sobre la corporalidad de las mujeres mapuche}

Otro de los enfoques planteados por la EPF, es el análisis de los impactos que la escasez hídrica tiene sobre la dimensión corporal (Sultana, 2011), ya que la dificultad de acceso al recurso vital genera una serie de desequilibrios corporales y psicoemocionales sobre las personas que lo padecen. En ese sentido, es importante vislumbrar cómo los roles que tiene cada individuo para acceder al recurso hídrico generará una expresión diferente en cada cuerpo.

Como se mencionó anteriormente, la labor de recepción y repartición de agua dentro del espacio domésticoes realizada en su mayoría por mujeres, situación que frente a condiciones de escasez hídrica implica un aumento de las distancias y de la cantidad de veces que se debe acudir a la búsqueda de agua. De manera que serían estas mujeres quienes encarnarían las mayores dificultades físico-emocionales. Sin embargo, en territorios de mayor concentración de población, pese a existir indicios de escasez, serían las condiciones tecnológicas, sumadas a la cooperación entre familiares, los aspectos que suavizarían los efectos de la escasez hídrica sobre los cuerpos de las mujeres mapuche habitantes de las Planicies Litorales. A continuación, analizamos tres principales categorías de impactos que ha tenido la escasez, y la consecuente búsqueda de agua en las mujeres mapuches entrevistadas.

En primer lugar, las mujeres más impactadas identificaron problemas corporales provocados principalmente a raíz del esfuerzo físico que deben realizar para gestionar el acceso y uso de agua. A partir de ello, se 
identificó que las actividades de traslado de agua, en carretas o baldes, entre largas distancias (señalan recorrer hasta 300 metros en busca de agua de río y/o vertiente), y a la cual deben acudir reiteradas veces durante la semana, les genera y acentúa dolores físicos sobre las extremidades y la columna vertebral. Sin embargo, debido a ser las únicas encargadas de la gestión del agua a nivel doméstico siguen ejerciendo dichas actividades.Por otra parte, además de recopilar el agua desde la fuente hasta el hogar, posteriormente aquellas que no tienen sistemas de distribución hidráulico dentro de este (un 76 \% de los encuestados), deben distribuir el agua de forma manual hacia las diferentes actividades que realicen, situación que además de generar impactos físicos, les generan impactos sobre el tiempo invertido en esta actividad -que se realiza de manera continua durante todo el día-.

Así se identifica que, si bien la estrategia de distribución hídrica desde fuentes de pozos, esteros y vertientes solía realizarse de manera manual, a partir de la escasez hídrica, estas actividades han generado fuertes dificultades por cambio de distancias recorridas (en territorio con altas pendientes) acortando los tiempos y las capacidades económicas. Además se observa que la entrega de agua encamión aljibe, si bien les permite paliar en parte el problema, se presenta como una forma de abastecimiento discontinuo, que debe necesariamente estar complementada con otras fuentes, por lo tanto, dependerá de la cercanía que cada mujer tenga a una fuente natural lo que deberá invertir en tiempo y esfuerzo físico para conseguir el agua.

En segundo lugar, se observaron impactos a nivel emocional, ya que la principal sensación de vulnerabilidad frente al problema de escasez, es la "incertidumbre e inseguridad" respecto a la disponibilidad futura de agua. Donde aquellas mujeres abastecidas por camión aljibe temen que la institucionalidad deje de suministrarles agua. Es importante destacar que el aislamiento es un aspecto importante de tomar en cuenta para diferenciar los impactos emocionales que la falta de disponibilidad de agua provocaría. Ya que dentro del relato de aquellas mujeres que deben hacerse cargo por si solas y en su mayoría de este aspecto, se observa una mayor preocupación y estrés al abordar este tema. Así lo expresa una de ellas cuando menciona:

"Yo sufro de agua. En enero ya me quedo sin agua. Eso fue porque plantaron muchos eucaliptos y se empezaron a secar los pozos. Y ahí tengo que ir a un arroyo a sacar agua con bidones y carreta.” (Mujer mapuche, 58 años)

Por ello, las entrevistadas coincidían en anhelar la recuperación de agua de sus pozos, por lo que mencionaron utilizaban una serie de estrategias de corta de vegetación exótica a cambio de reforestación de vegetación nativa. Así lo narra una entrevistada:

"Yo llegué de 9 años a Bahía Mansa, y cuando empezaron las plantaciones empecé a ver que las aguas empezaron a escasear. Yo lo vi en mi terreno, donde para hacer mi casa tuve que sacar los eucaliptos que habían plantado, y el terreno ya estaba seco y ahora este terreno no es bueno para la huerta porque la tierra se parte, porque no tiene agua” (Mujer mapuche, 62 años)

Del mismo modo, mencionaron como estrategias que les alivian y dan confianza la recopilación de aguas lluvias, ya que mencionan es una fuente que pueden recepcionar cerca del hogar y perciben limpia, la que almacenan y en algunos casos hierven para beber. Sin embargo, esta dinámica depende del periodo de lluvia por lo que se utiliza principalmente como una fuente complementaria confiable. Es importante destacar la presión que la escasez hídrica genera al aumentar la actividad mental por la búsqueda de agua, tomando en cuenta que la labor doméstica ya requiere gran cantidad de atenciones mentales, al faltar el agua, estas aumentarán, generando mayores niveles de estrés y preocupación en las mujeres afectadas.

Finalmente, se evidenció que la mayoría de las entrevistadas le atribuyen un valor biológico vital al agua, el cual consideran necesario para llevar a cabo todas las actividades que realizan. Algunas de ellas mencionaron que el agua, además de ser un elemento vital, es parte del territorio (wallmapu) que la cosmovisión mapuche concibe como un todo y cuyo valor va siendo transmitido de generación en generación, el que se ve quebrantado al ser conceptualizado como un elemento económico desde la visión hegemónica occidental. Con ello, explican que dentro de su cosmovisión, el agua presenta una fuerza o espíritu propio, que denominan "ngen-ko" (donde "ngen" es fuerza y "ko" es agua), el cual es pasado a llevar a través de su descuido. Por lo tanto, es importante poner en conocimiento cómo las diferencias culturales generarán concepciones diversas 
respecto a la relación de las sociedades y la naturaleza. En este caso, es posible visibilizar que a la vez que la falta de disponibilidad de agua impacta sobre los modos de vida cotidianos de la población más vulnerable, también generaría un impacto sobre la espiritualidad del Pueblo Mapuche.

\section{Discusión}

En esta investigación demostramos de qué manera se afectan las vidas cotidianas de mujeres mapuche rurales en un territorio donde las fuentes de agua y los tipos de abastecimiento hídrico en los hogares se están viendo afectados, producto de una disminución en la disponibilidad de agua. El análisis de las medidas proporcionadas por las instituciones encargadas, deja ver que las soluciones de carácter urgente terminan siendo permanentes, generando considerables impactos en la vida cotidiana y el bienestar de las personas a cargo de la gestión hídrica doméstica. Tal es el caso de la entrega de agua a través de camiones aljibes, solución que si bien responde a las primeras necesidades de la población, les quita autonomía, las hace dependientes de agua externa a sus territorios y potencia el asistencialismo institucional. Por lo tanto, se revela la importancia de la propuesta de la EPF en ahondar en las micropolíticas que influyen en la toma de decisiones a nivel doméstico para acceder, usar y proteger el recurso hídrico (Truelove, 2011). De esta manera, es posible reconocer que a la vez que la población se ve fuertemente impactada, es capaz de proponer estrategias de acceso y uso, y revalorizar el recurso en escasez, como se constató con la práctica de almacenamiento de aguas lluvias, y la reutilización de agua entre actividades. Por otra parte, cabe destacar que omitiendo la visión microescalar se pierden de vista los usos que se le da al agua, y de esta manera, se borra la importancia que esta tiene para solventar y desarrollar las tradiciones de los territorios.

Otra aplicación importante que aporta la propuesta de la EPF al análisis de problemas socioambientales, tiene que ver con la des-homogenización de los territorios (Sultana, 2015). Ya que, al indagar sobre las prácticas locales, y en particular domésticas, es posible tener una visión más precisa de la realidad hídrica de los usuarios, según su raza, género, y clase social. Así se demuestra en el estudio, donde a través de la comparación de las dinámicas domésticas de mujeres de la Cordillera de la Costa y las Planicies Litorales, fue posible identificar que si bien, las mujeres cumplen un importante rol en las decisiones hídricas a nivel doméstico, los impactos que encarnan son diferentes por cuestiones geográficas.

Ahora bien, retomando la noción del género y la cultura como condiciones que definen roles (Lamas, 2000), sigue siendo importante destacar, que dentro de la cultura heteropatriarcal, es imperante visibilizar labores que históricamente han sido escondidas del ojo público. Por ello, el análisis de la escala doméstica, a la vez que permite conocer quién, cómo, y dónde sufre de escasez hídrica, permite dar relevancia a los trabajos de sostenibilidad de la vida -vinculados directamente al agua- que han realizado en su mayoría las mujeres, de manera de comprender experiencias benéficas y dificultades, para, desde esa visión proponer políticas de género, que se vinculen a lo que efectivamente se vive en el espacio doméstico.Lo mismo es aplicable a las diferencias culturales encarnadas en los individuos; al homogeneizar, y a la vez invisibilizar, las diferencias culturales, se ignora/excluye la cosmovisión de culturas ancestrales como la cultura mapuche, generando así la pérdida de aspectos identitarios claves de la misma. Llegando a difuminar la espiritualidad ligada al respeto de la naturaleza, y las tradiciones históricas vinculadas a esta relación. Por otra parte, se destaca el análisis de la dimensión corporal y emocional (Sultana, 2015) que propone la EPF, que permite ver impregnados en la experiencia particular de las personas, los dolores, las emociones, y las dificultades que las desigualdades hídricas generan en los cuerpos, y que han sido abordados de manera despersonalizada a través del estudio de las generalidades territoriales.

Se concluye a través de esta investigación, que es relevante dentro de la disciplina geográfica el enfoque en las particularidades, subjetividades y microescalaridades propuestas por la EPF, ya que la construcción de las teorías del ambiente se han basado en la proposición de un conocimiento científico que se posiciona como autónomo, objetivo y neutral (Vizcarra, 2005), razón por la que se observan y posicionan algunas temáticas por sobre otras (tal es el caso del espacio público, por sobre el doméstico). Esto, generó dentro de los estudios del territorio un sesgo que ha dejado de lado ciertas dimensiones -identitarias y particulares de las y los individuosque construyen de igual manera la realidad. 
Finalmente, se destaca el valor que esta investigación tiene para los futuros análisis hídricos con perspectivas feministas en Chile. Ya que si bien, el territorio cuenta con amplia literatura que aborda los efectos socioterritoriales de la escasez hídrica, poco abordadas han sido las prácticas de acceso y uso de agua y el impacto doméstico que esta problemática genera en los territorios. Aspectos que, de ser abordados, podrían aportar a la efectividad y coherencia de las decisiones tomadas a nivel central con las vivencias locales particulares de las y los habitantes.

\section{Agradecimientos}

Las autoras agradecen a todas las mujeres que participaron del estudio, y compartieron sus opiniones y experiencias. La presente investigación recibió apoyos financieros del Proyecto Anillo, SOC1404: Dinámicas naturales, espaciales y socio-culturales: perspectivas sobre los conflictos socio-ambientales en territorios forestales de Chile,1975-2014.

\section{Bibliografía}

Barlow, M. (2008).El convenio azul: La crisis global del agua y la batalla futura por el derecho al agua.Santiago, Chile: Heinrich Bo?ll Stiftung, Cono Sur

Bartra, Eli (2002). Reflexiones Metodológicas en Debates en torno a una metodología feminista. Ciudad de México, México: UAM, UNAM

Berkes, F. (2010). Devolution of environment and resources governance: trends and future. Winnipeg, Canada: Natural Resources Institute - University of Manitoba.

Biblioteca Del Congreso Nacional (s/f).Relieve Región de los Lagos. Recuperado de https://www.bcn.cl/siit/ nuestropais/region10/relieve.htm

Biblioteca Nacional Del Congreso Nacional (2015). Emergencia Agrícola 2015 por los efectos de la Sequía. Recuperado de https://www.bcn.cl/siit/actualidad-territorial/emergencia-agricola-2015por-los-efectos-de-lasequia/document_view2

Boelens, R. y Zwarteveen, M. (2014).Defining, researching and struggling for water justice: some conceptual building blocks for research and action, Water International, 39 (2), 143-158

Brooks, D. \& Wolfe, Sarah (2003). Water scarcity: An alternative view and its implications for policy and capacity building. Natural Resources Forum (27), 99-107. Recuperado de: https://www.sarahwolfe.ca/wp-content/ uploads/2011/06/finalwolfebrooks.pdf

Budds, J., y Hinojosa, L., (2012). Las Industrias Extractivas y los Paisajes hídricos en transición en los Países andinos: análisis de la gobernanza de recursos y formación de territorios en Perú.En Isch, E., Boelens, R., y Peña, F. (eds). Agua, Injusticia y Conflictos. Lima, Peru: CBC.

Camus, P. (2006). Ambiente, Bosques y gestión forestal en Chile 1541 - 2005. Santiago, Chile: Centro de Investigaciones Barros Arana de la Dirección de Bibliotecas, Archivos y Museos -Lom Ediciones

Castro, H. (2010). Conflictos por el agua en Chile: Entre los derechos humanos y las reglas del mercado.Santiago, Chile: Programa Chile Sustentable. hile

Catalán, R., y Ramos, R., (1999). Pueblo Mapuche, Bosque Nativo y Plantaciones Forestales: Las causas Subyacentes de la deforestación en el Sur de Chile. Temuco, Chile: Universidad Católica de Temuco.

Conferencia Internacional sobre el Agua y el Medio Ambiente (1992). La mujer y la gestión de los recursos 
hídricos: un enfoque integrado. Principio 3 de Dublín. Dublín.

Cruz, M.E. y Rivera, R. (1983). La realidad Forestal Chilena. Grupo de Investigaciones Agrarias. Santiago, Chile: Universidad Academia de Humanismo Cristiano.

Diaz, L., Torruco,U., Martinez, M. Y Varela, M. (2013). La entrevista, recurso flexible y dinámico.Ciudad de México, México: Universidad Nacional Autónoma de México.

Dirección General de Aguas (2004). Diagnóstico y clasificación de los cursos y cuerpos de agua según objetivos de calidad: Cuenca del Río Bueno.

Doria, M. (2006). Bottled water versus tap water: understanding consumers preferences. Norwich, UK: University of East Anglia

Figueroa, P. (2014). Variabilidad de las precipitaciones (1965-2012) en Chile centro- sur $\left(33,9^{\circ} \mathrm{S}-41,6^{\circ} \mathrm{S}\right)$ mediante índices estadísticos y temporales de la irregularidad. Memoria Pregrado. Santiago, Chile:Universidad de Chile.

Frêne, C., G. Ojeda, J. Santibáñez, C. Donoso, J. Sanzana, C. Molina, P. Andrade y M. Núñez-Ávila. (2014). Agua en Chile: diagnósticos territoriales y propuestas para enfrentar la crisis hídrica73 p

Fundación Amulén (2019). Pobres de agua. Radiografía del agua rural de Chile: Visualización de un problema oculto. Recuperado de https://static1.squarespace.com/static/5afc52595ffd20cbdc764a27/t/ 5cf84bdaab32aa0001d08a08/1559776320566/Informe_Amulen.pdf

Gutierrez, R.; Rivera, A.: Vela, M.; Bravo, Y.; (2007). El agua y el desarrollo rural. Ciudad de México, México:Centro de Estudios para el Desarrollo Rural Sustentable y la soberanía alimentaria.

Herrero, Y., y Pascual, M., (2010). Ecofeminismo, una propuesta para repensar el presente y construir el futuro. Madrid, España: Centro Nacional de Educación Ambiental.

Herrero, Y. (2016). Economía feminista y economía ecológica, el dialogo necesario y urgente. Revista de Economía Crítica, (22) 144-161. Recuperado de http://revistaeconomiacritica.org/sites/default/files/revistas/ Revista_Economia_Critica_22.pdf

Ilustre Municipalidad de San Juan de La Costa (2012). Actualización plan de desarrollo comunal (PLADECO) 2012 - 2017. San Juan de la Costa, Chile

Instituto Nacional de Estadísticas (2007). Censo Agropecuario y Forestal 2007. Recuperado de http://www.ine.cl/ estadisticas/censos/censo-agropecuario-y-forestal-2007

Johnston, B. (2003). The Political Ecology of Water: An Introduction. Capitalism Nature Socialism14. 73-90. doi: 10.1080/10455750308565535.

Lamas, M. (2000). Diferencias de sexo, género y diferencia sexual.Cuicuilco , 7 (18) Recuperado de https:// www.redalyc.org/pdf/351/35101807.pdf

Lara, A., Little, C., McPhee, J., Urrutia, R. (2015).Revealing the impact of forest exotic plantations on water yield in large scale watersheds in South-Central Chile. Valdivia, Chile: Universidad Austral de Chile.

Larraín, S. (2006). El agua en Chile: entre los derechos humanos y las reglas del mercado. Polis Revista Latinoamericana (14). Recuperado de: http://polis.revues.org/5091 
Leff, E. (2003). La ecología política en América Latina: un campo en construcción. Polis Revista Latinoamericana (5). Recuperado de: http://journals.openedition.org/polis/6871

Linton, J. (2014). Modern water and its social discontents: a history of hydrosocialrenewal. WileyInterdisciplinary Reviews: Water(1),13-43.doi:10.1002/wat2.1009

Mullee, M, y Woodhouse, P. (2017). Water Governance: An Historical Perspective on Current Debates. World Development92, 225-241.

Martinez, J. (2010).Judith Buttler: Cuerposqueimportan. Sobre los límites materiales y discursivos del «sexo».Revista Chilena de Investigaciones Estéticas, (52),497-500.Recuperado de https://scielo.conicyt.cl/pdf/aisthesis/ n52/art27.pdf

Mehta, L. (2006). Whose scarcity? Whose property? The case of water in western India. Institute of Development Studies, University of Sussex, Brighton, UK

(2003). Contexts and Constructions of Water Scarcity. Source: Economic and PoliticalWeekly.Economic and political weekly,38(48).doi:10.2307/4414344

ONU (2003). Agua para todos, agua para la vida. Informe de las Naciones Unidas sobre el Desarrollo de los Recursos Hídricos en el Mundo. Recuperado de https://unesdoc.unesco.org/ark:/48223/pf0000149406

FAO (2013). Afrontar la escasez de agua Un marco de acción para la agricultura y la seguridad alimentaria. 38. Informe sobre temas hídricos. Recuperado de http://www.fao.org/3/a-i3015s.pdf

Otero, L. (1997). Efectos ambientales del reemplazo de bosque nativo por plantaciones Estudio en cuatro microcuencas en la provincia de Valdivia.Ciencia E investigación Forestal, 8(2),253-276.Recuperado de https://bibliotecadigital.infor.cl/bitstream/handle/20.500.12220/10862/18560.pdf

Padilla, E. (2012). Construcción Social de la escasez de agua. Una perspectiva teórica anclada en la construcción territorial. Centro de estudios Históricos de Región y Frontera de El Colegio de Sonora,24 (3) 91-116 .Recuperado de http:/www.scielo.org.mx/scielo.php?script=sci_arttext\&pid=S1870-39252012000600004

Pérez, A. (2014). Subversión feminista de la economía. Aportes para un debate sobre el conflicto capital-vida. Madrid, España: Editorial Traficantes de sueños.

Prieto, M. (2015). Ecología política del Agua. En Ecología Política en Chile: naturaleza, propiedad, conocimiento y poder.Santiago, Chile: Editorial Universitaria.

Quilaqueo, F. (2013). Mujer Mapuche, Historia, persistencia y continuidad.Santiago, Chile: Editorial Icaria.

Rico Amorós, A.M., Olcina Cantos, J., \& Saurí, D. (2009). Tourist land use patterns and water demand: Evidence from the Western Mediterranean.Land Use Policy, 26(2), 493-501.doi:10.1016/j.landusepol.2008.07.002

Rico, M. (1998). Las mujeres en los procesos asociados al agua en America Latina. Estado de situación, propuestas de investigación y de políticas. Unidad Mujer y Desarrollo, CEPAL.Recuperado de https:// repositorio.cepal.org/bitstream/handle/11362/19798/S9810813_es.pdf

Rijsberman, F. (2006). Water scarcity: Fact or fiction?.Agricultural Water Management,80(1-3), 5-22.doi: 10.1016/ j.agwat.2005.07.001

Rocheleau, D., Thomas-Slayter, B., Wangari, E. (1995). Género y Ambiente: Una perspectiva de la ecología política feminista.México.D.F, México 
Santana C., Nancy (2006). El Ecofeminismo Latinoamericano. Las Mujeres y la Naturaleza como Símbolos.Trujillo, Perú: Universidad de los Andes.

Shiva, V., Flores, J., Martinez, E. (2012). Ecofeminismo desde los derechos de la naturaleza. Recuperado de https:/ /generoymineriaperu.files.wordpress.com/2013/10/ecofem-ecuador1.pdf

Stratford, E. (1995) Gender and Environment: Some preliminary questions about women and water in the South Australian context, Gender, Place \& Culture: A Journal of Feminist Geography, 2 (2) 209-216

Sultana, F. (2011). Suffering for water, suffering from water: Emotional geographies of resource access, control and conflict. Geoforum,42(2),163-172.doi 10.1016/j.geoforum.2010.12.002

(2014). Gendering Climate Change: Geographical Insights. The Professional Geographer, 66(3), 372381. doi: 10.1080/00330124.2013.821730

(2015). Rethinking Community and Participation in Water Governance. In The Routledge Handbook of Gender and Development(pp.261-272).ReinoUnido: Routledge Taylor \& Francis Group

Swyngedouw, E. (2009). The Political Economy and Political Ecology of the Hydro-Social Cycle. Journal of contemporary water research \& education, (142), 56-60.doi:10.1111/j.1936-704X.2009.00054.x

Truelove, Y. (2011). (Re)Conceptualizing wáter inequality in Delhi, Ingia Through a feminist political ecology framework.Geoforum,(42),143-152.doi:10.1016/j.geoforum.2011.01.004

Vargas, J, L. (2016). El territorio mapuche de Mariküga frente al modelo forestal.En Resistencias Mapuche al extractivismo (pp.17-28).Santiago,Chile:Editorial Mapuexpress.

Villarroel, C. (2012). Asociaciones comunitarias de Agua Potable Rural en Chile: Diagnósticos y Desafíos. Santiago, Chile: Grafica Andes Ltda

Vizcarra, (2005). El saber ecológico local femenino en Ciencia, tecnología y género en Iberoamérica. Ciudad de México, México: Centro de investigaciones interdisciplinarias en Ciencias y Humanidades. Universidad Nacional autónoma de México. 